\title{
Geometric series of numbers approximating positive integers
}

\author{
Martin W. Bredenkamp \\ Department of Science, Asia-Pacific International University, PO Box 4, MuakLek, Saraburi Province, 18180, Thailand \\ Email address: \\ martinb@apiu.edu
}

\section{To cite this article:}

Martin W. Bredenkamp. Geometric Series of Numbers Approximating Positive Integers, Pure and Applied Mathematics Journal. Vol. 2, No. 2, 2013, pp. 79-93. doi: 10.11648/j.pamj.20130202.15

\begin{abstract}
The predictability of cycles in the series of Pythagorean triples led to an investigation that yielded numbers $(x)$ that are associated with irrational square roots $(\sqrt{n})$. The cycles recur with geometric factors (cycle factors $y$ ) that are comprised of a positive integer $x$ where $y=x+\sqrt{x^{2} \pm 1}$. On raising the cycle factors to the positive integer powers $\left(y^{m}\right)$, a series is generated where each consecutive member comes closer and closer to positive integers as the series progresses. A formula associates the square root $(\sqrt{n})$ with these series. Prime factorising the positive integers in the power series $\left(x_{m}\right)$ produces predictable patterns among the prime factors in the series. In general, power series that have each consecutive member in the series come closer to positive integers are limited to $\left(x+\sqrt{x^{2} \pm r}\right)^{m}$ where $x$ and $r$ are positive integers and $r$ $<(x+1)^{2}-x^{2}$ for the $+r$ condition and $r<x^{2}-(x-1)^{2}$ for the $-r$ condition.
\end{abstract}

Keywords: Power Series of Irrational Numbers, Approximating Positive Integers, Factors That Relate Perfect Squares, Prime Factor Patterns

\section{Introduction}

In the previous paper [1], we reported series of rightangled triangles which provide series of rational numbers that have as their limits irrational square roots. A fascinating aspect of all these series, is the constants that are generated and the predictability of these series. We were attracted to the geometric nature of the recurrence of these phenomena, which are especially noticeable at the series with many subseries. The indices [2] of the triangles, and thus the triangles, recur with geometric regularity. This paper deals with a search into the nature of the recurrence of these series in an effort to rationalise this phenomenon.

\section{The Cycle Factor of $\sqrt{2}$}

The place to begin this investigation is with the series of rational numbers that leads to $\sqrt{2}$. Table 1 shows the indices [2] and the sides of the series of triangles that lead to the $45^{\circ}$ triangle. The ratio of $i_{n+1} / i_{n}$ and $j_{n+1} / j_{n}$ are also provided for investigation. Both these ratios are converging to the same number, which we term the cycle factor $(y)$. As the number develops in the table the part that remains unchanged and is the same as the limit is underlined. The li-mit is irrational since it is $1+\sqrt{2}$ [1]. These converging ratios alternate between being greater than, and less than the limit. The $i$ - and the $j$-series are out of phase in their alternations.

The cycle factor puzzled us, and we tried to determine its significance. We recorded it and looked at the cycle factors of the greater roots.

\section{Cycle Factors (y) of the Roots of Numbers Adjacent to Perfect Squares}

There are two series that lead to $\sqrt{3}$, its $e$-series, where the even-numbered leg of the series of triangles is approaching the irrational leg of the limiting triangle ( $\sqrt{3} / 1 / 2$-triangle), and the $u$-series where the uneven numbers approach the irrational leg of the same triangle. Both these series have the same indices, the only difference being that the $i$-indices of the $e$ - and $u$-series are dislocated by one position. The quotient of the indices will yield the same result. Just as with $\sqrt{2}$ where the cycle factor is the sum of an integer and a square root, the cycle factor for $\sqrt{3}$ is $2+\sqrt{3}$. It turns out that the cycle factors of all the numbers just short of or just greater than a perfect square is comprised of that square root plus the square root $(x)$ of the adjacent perfect square, irrespective of what triangle series is used to obtain the cycle factor of that square root. Table 2 
contains the cycle factors of all the numbers adjacent to a perfect square up to 101 .

Table 1. The series of triangles $(i, j)_{n}$ that have $h / e$ and $h / u$ converge to $\sqrt{\mathbf{2}}$, and the cycle factors of their indices.

\begin{tabular}{|c|c|c|c|c|c|c|c|}
\hline$n$ & $i$ & $i$ & $u$ & $e$ & $\boldsymbol{h}$ & $i_{n+1} / i_{n}$ & $\mathbf{j}_{\mathbf{n}+1} / \mathbf{j}_{n}$ \\
\hline 1 & 1 & 2 & 3 & 4 & 5 & 3 & $\underline{2}$ \\
\hline 2 & 3 & 4 & 21 & 20 & 29 & 2.33333333333333 & 2.5 \\
\hline 3 & 7 & 10 & 119 & 120 & 169 & 2.42857142857143 & 2.4 \\
\hline 4 & 17 & 24 & 697 & 696 & 985 & 2.41176470588235 & 2.41666666666667 \\
\hline 5 & 41 & 58 & 4059 & 4060 & 5741 & 2.41463414634146 & 2.41379310344828 \\
\hline 6 & 99 & 140 & 23661 & 23660 & 33461 & 2.41414141414141 & 2.41428571428571 \\
\hline 7 & 239 & 338 & 137903 & 137904 & 195025 & 2.41422594142259 & 2.41420118343195 \\
\hline 8 & 577 & 816 & 803761 & 803760 & 1136689 & 2.41421143847487 & 2.41421568627451 \\
\hline 9 & 1393 & 1970 & 4684659 & 4684660 & 6625109 & 2.41421392677674 & 2.41421319796954 \\
\hline 10 & 3363 & 4756 & 27304197 & 27304196 & 38613965 & 2.41421349985132 & 2.41421362489487 \\
\hline 11 & 8119 & 11482 & 159140519 & 159140520 & 225058681 & 2.41421357310013 & 2.41421355164605 \\
\hline 12 & 19601 & 27720 & 927538921 & 927538920 & 1311738121 & 2.41421356053263 & 2.41421356421356 \\
\hline 13 & 47321 & 66922 & 5406093003 & 5406093004 & 7645370045 & 2.41421356268887 & 2.41421356205732 \\
\hline 14 & 114243 & 161564 & 31509019101 & 31509019100 & 44560482149 & 2.41421356231892 & 2.41421356242727 \\
\hline 15 & 275807 & 390050 & 183648021599 & 183648021600 & 259717522849 & \multicolumn{2}{|c|}{$1+\sqrt{2}=2.414213562373095048801689$} \\
\hline
\end{tabular}

Table 2. Cycle factors of roots of numbers adjacent to perfect squares.

\begin{tabular}{rllllll}
\hline \multirow{2}{*}{ Root } & \multicolumn{2}{l}{ Cycle factor } & & \multicolumn{3}{l}{ Cycle factor } \\
\cline { 2 - 5 } & radical & decimal & & radical & decimal \\
\hline$\sqrt{2}$ & $1+\sqrt{2}$ & 2.41421356237309504880 & $\sqrt{37}$ & $6+\sqrt{37}$ & 12.0827625302982196890 \\
$\sqrt{3}$ & $2+\sqrt{3}$ & 3.73205080756887729353 & $\sqrt{48}$ & $7+\sqrt{48}$ & 13.9282032302755091741 \\
$\sqrt{5}$ & $2+\sqrt{5}$ & 4.23606797749978969641 & $\sqrt{50}$ & $7+\sqrt{50}$ & 14.0710678118654752440 \\
$\sqrt{8}$ & $3+\sqrt{8}$ & 5.828427124746619009760 & $\sqrt{63}$ & $8+\sqrt{63}$ & 15.9372539331937717715 \\
$\sqrt{10}$ & $3+\sqrt{10}$ & 6.16227766016837933200 & $\sqrt{65}$ & $8+\sqrt{65}$ & 16.0622577482985496524 \\
$\sqrt{15}$ & $4+\sqrt{15}$ & 7.87298334620741688517 & $\sqrt{80}$ & $9+\sqrt{80}$ & 17.9442719099991587856 \\
$\sqrt{17}$ & $4+\sqrt{17}$ & 8.12310562561766054982 & $\sqrt{82}$ & $9+\sqrt{82}$ & 18.0553851381374166266 \\
$\sqrt{24}$ & $5+\sqrt{24}$ & 9.89897948556635619639 & $\sqrt{99}$ & $10+\sqrt{99}$ & 19.94988743710661995473 \\
$\sqrt{26}$ & $5+\sqrt{26}$ & 10.0990195135927848300 & $\sqrt{101}$ & $10+\sqrt{101}$ & 20.0498756211208902702 \\
$\sqrt{35}$ & $6+\sqrt{35}$ & 11.9160797830996160426 & & & \\
\hline
\end{tabular}

\section{All Other Cycle Factors (y)}

Having established the cycle factors of the roots of the numbers one greater or one less than a perfect square, is it possible to determine the cycle factors of all the other numbers? An investigation into the cycle factors in table 2 shows that the numbers are close to an even positive integer, because one component is an integer and the other the square root of a value very close to the square of that same integer. Studying the approximate cycle factors of the roots of numbers not adjacent to a perfect square as determined by the quotient of indeces as done previously for $\sqrt{2}$, we find that these cycle factors are also close to even integers. Taking the cue, we find that the approximate cycle factors are very close to the sum of the square root of a perfect square and the square root of the number just larger or just 
smaller than that perfect square (table 3), which turns out to be the actual cycle factors.

Table 3. The exact and calculated cycle factors of the roots less than the square root of 102.

\begin{tabular}{|c|c|c|c|c|c|c|c|}
\hline \multirow{3}{*}{ Root } & \multicolumn{3}{|l|}{ Cycle factor } & \multirow{3}{*}{ Root } & \multicolumn{3}{|l|}{ Cycle factor } \\
\hline & \multirow{2}{*}{ radical } & \multicolumn{2}{|l|}{ decimal } & & \multirow{2}{*}{ radical } & \multicolumn{2}{|l|}{ decimal } \\
\hline & & exact & index $_{n} /$ index $_{n-1}$ & & & exact & index $_{n} /$ index $_{n-1}$ \\
\hline$\sqrt{2}$ & $1+\sqrt{2}$ & 2.41421356237 & 2.41421356243 & $\sqrt{53}$ & $182+\sqrt{33125}$ & 364.002747232 & 364.002747228 \\
\hline$\sqrt{3}$ & $2+\sqrt{3}$ & 3.73205080757 & 3.73205080758 & $\sqrt{55}$ & $89+\sqrt{7920}$ & 177.994381845 & 177.994381613 \\
\hline$\sqrt{5}$ & $2+\sqrt{5}$ & 4.23606797750 & 4.23606797748 & $\sqrt{56}$ & $15+\sqrt{224}$ & 29.9666295471 & 29.9666294756 \\
\hline$\sqrt{7}$ & $8+\sqrt{63}$ & 15.9372539332 & 15.9372539327 & $\sqrt{57}$ & $151+\sqrt{22800}$ & 301.996688705 & 301.997183099 \\
\hline$\sqrt{8}$ & $3+\sqrt{8}$ & 5.82842712475 & 5.82842712464 & $\sqrt{58}$ & $\begin{array}{l}19603+ \\
\sqrt{384277608}\end{array}$ & 39205.9999745 & 39205.9999743 \\
\hline$\sqrt{10}$ & $3+\sqrt{10}$ & 6.16227766017 & 6.16227766011 & $\sqrt{59}$ & $530+\sqrt{280899}$ & 1059.99905660 & 1059.99920723 \\
\hline$\sqrt{11}$ & $10+\sqrt{99}$ & 19.9498743711 & 19.9498743711 & $\sqrt{61}$ & $\begin{array}{l}29718+ \\
\sqrt{883159525}\end{array}$ & 59436.0000168 & 59436.0000140 \\
\hline$\sqrt{13}$ & $18+\sqrt{325}$ & 36.0277563773 & 36.0277563765 & $\sqrt{63}$ & $8+\sqrt{63}$ & 15.9372539332 & 15.9372539332 \\
\hline$\sqrt{15}$ & $4+\sqrt{15}$ & 7.87298334621 & 7.87298334438 & $\sqrt{65}$ & $8+\sqrt{65}$ & 16.0622577483 & 16.0622577483 \\
\hline$\sqrt{17}$ & $4+\sqrt{17}$ & 8.12310562562 & 8.12310562666 & $\sqrt{67}$ & $\begin{array}{l}48842+ \\
\sqrt{2385540963}\end{array}$ & 97683.9999898 & 97683.9999897 \\
\hline$\sqrt{19}$ & $170+\sqrt{28899}$ & 339.997058798 & 339.997059744 & $\sqrt{69}$ & $7775+\sqrt{60450624}$ & 15549.9999357 & 15549.9999347 \\
\hline$\sqrt{21}$ & $55+\sqrt{3024}$ & 109.990908339 & 109.990908288 & $\sqrt{71}$ & $3480+\sqrt{12110399}$ & 6959.99985632 & 6959.99985553 \\
\hline$\sqrt{23}$ & $24+\sqrt{575}$ & 47.9791576166 & 47.9791576106 & $\sqrt{72}$ & $17+\sqrt{288}$ & 33.9705627485 & 33.9705627484 \\
\hline$\sqrt{24}$ & $5+\sqrt{24}$ & 9.89897948557 & 9.89897948448 & $\sqrt{73}$ & $1068+\sqrt{1140625}$ & 2136.00046816 & 2136.00054259 \\
\hline$\sqrt{26}$ & $5+\sqrt{26}$ & 10.0990195136 & 10.0990195127 & $\sqrt{74}$ & $3699+\sqrt{13682600}$ & 7397.99986483 & 7397.99983509 \\
\hline$\sqrt{27}$ & $26+\sqrt{675}$ & 51.9807621135 & 51.9807621096 & $\sqrt{75}$ & $26+\sqrt{675}$ & 51.9807621135 & 51.9807621136 \\
\hline$\sqrt{29}$ & $70+\sqrt{4901}$ & 140.007142493 & 140.007142389 & $\sqrt{77}$ & $351+\sqrt{123200}$ & 701.998575496 & 701.998604002 \\
\hline$\sqrt{31}$ & $1520+\sqrt{2310399}$ & 3039.99967105 & 3039.99919808 & $\sqrt{79}$ & $80+\sqrt{6399}$ & 159.993749756 & 159.993749763 \\
\hline$\sqrt{32}$ & $17+\sqrt{288}$ & 33.9705627485 & 33.9705627485 & $\sqrt{80}$ & $9+\sqrt{80}$ & 17.9442719100 & 17.9442719100 \\
\hline$\sqrt{33}$ & $23+\sqrt{528}$ & 45.9782505862 & 45.9782505792 & $\sqrt{82}$ & $9+\sqrt{82}$ & 18.0553851381 & 18.0553851397 \\
\hline$\sqrt{34}$ & $35+\sqrt{1224}$ & 69.9857113691 & 69.9857113487 & $\sqrt{83}$ & $82+\sqrt{6723}$ & 163.993902212 & 163.993902206 \\
\hline$\sqrt{35}$ & $6+\sqrt{35}$ & 11.9160797831 & 11.9160797828 & $\sqrt{85}$ & $378+\sqrt{142885}$ & 756.001322749 & 756.001347388 \\
\hline$\sqrt{37}$ & $6+\sqrt{37}$ & 12.0827625303 & 12.0827625306 & $\sqrt{87}$ & $28+\sqrt{783}$ & 55.9821371593 & 55.9821370945 \\
\hline$\sqrt{39}$ & $25+\sqrt{624}$ & 49.9799919936 & 49.9799919892 & $\sqrt{88}$ & $197+\sqrt{38808}$ & 393.997461913 & 393.997542998 \\
\hline$\sqrt{40}$ & $19+\sqrt{360}$ & 37.9736659610 & 37.9736659366 & $\sqrt{89}$ & $500+\sqrt{250001}$ & 1000.00100000 & 1000.00100431 \\
\hline$\sqrt{41}$ & $32+\sqrt{1025}$ & 64.0156211872 & 64.0156211884 & $\sqrt{91}$ & $1574+\sqrt{2477475}$ & 3147.99968234 & 3147.99968315 \\
\hline$\sqrt{43}$ & $\begin{array}{l}3482+ \\
\sqrt{12124323}\end{array}$ & 6963.99985640 & 6963.99966113 & $\sqrt{93}$ & $\begin{array}{l}12151+ \\
\sqrt{147646800}\end{array}$ & 24301.9999589 & 24301.9999592 \\
\hline$\sqrt{45}$ & $161+\sqrt{25920}$ & 321.996894380 & 321.997128087 & $\sqrt{95}$ & $39+\sqrt{1520}$ & 77.9871773792 & 77.9871774124 \\
\hline$\sqrt{47}$ & $48+\sqrt{2303}$ & 95.9895822028 & 95.9895806861 & $\sqrt{96}$ & $49+\sqrt{2400}$ & 97.9897948557 & 97.9897948555 \\
\hline$\sqrt{48}$ & $7+\sqrt{48}$ & 13.9282032303 & 13.9282032302 & $\sqrt{97}$ & $5604+\sqrt{31404817}$ & 11208.0000892 & 11208.0000872 \\
\hline$\sqrt{50}$ & $7+\sqrt{50}$ & 14.0710678119 & 14.0710678119 & $\sqrt{99}$ & $10+\sqrt{99}$ & 19.9498743711 & 19.9498743607 \\
\hline$\sqrt{51}$ & $50+\sqrt{2499}$ & 99.9899989998 & 99.9899976739 & $\sqrt{101}$ & $10+\sqrt{101}$ & 20.0498756211 & 20.0498756294 \\
\hline
\end{tabular}

Glancing through the data, several aspects are easily noticed.

- The cycle factors seem to be quite random. The larger the cycle factor, the closer it is to a positive integer. The largest cycle factor is that of $\sqrt{67}$ and its decimals begin with four $9 \mathrm{~s}$.

- Most of them are just less than a positive integer, but a few are just larger than a positive integer.

- The powers of ten seem to be present as cycle factors, at least the first three. $\sqrt{24}$ and $\sqrt{26}$ are just below and above 10 , respectively, $\sqrt{51}$ is just below 100 , and $\sqrt{89}$ is just above 1000 .

- In odd places, cycle factors are the same, such as for $\sqrt{7}$ and $\sqrt{63}$, which both have a cycle factor of 15.937253933. What do 7 and 63 have in common? $7 \times 9=63$. Likewise $\sqrt{11}$ and $\sqrt{99}$ have the same cycle factors. Once again 11 is a factor of 99.63 and 99 are parts of the $9 \times$ table. 
- Some of the index-derived approximations to the cycle factors are exactly the same as the exact cycle factors up to the 12 significant figures provided in the table, eg $\sqrt{32}$ and $\sqrt{50}$. On the other hand, some of the greater cycle factors vary at the last 7 of the 12 significant figures, because of limited indeces eg $\sqrt{45}$ and $\sqrt{57}$.

If table 2 were to be extended, all of the cycle factors of the square roots of numbers not adjacent to perfect squares in table 3 would be incorporated. We see therefore that the cycle factors of the square roots of numbers not adjacent to perfect squares make "premature" use of the cycle factors of square roots of numbers adjacent to perfect squares. What do they have in common? At the fourth bullet above we observed that numbers whose square roots share a cycle factor have the smaller number a factor of the larger. Let us look at a few more and observe this phenomenon closer. For $\sqrt{21}$ the cycle factor is $55+\sqrt{3024} .21$ is a factor of 3024. For $\sqrt{69}$ the cycle factor is $7775+\sqrt{60450624} .69$ is a factor of 60450624 . A look at the complementing factor reveals an important fact. For 7 and 63, whose roots share a cycle factor, the complementing factor is 9 , likewise for 11 and 99 (bullet four above). For 21 and 3024 the complementing factor is 144 and for 69 and 60450624 the complementing factor is 876096. All three these complementing factors are perfect squares $(9-3,144-12$ and $876096-936)$. We therefore have a relationship $n \times z^{2}$ $=t$, where $t$ is the number adjacent to a perfect square, $n$ is a smaller number whose root shares the cycle factor with the root of $t$, and $z^{2}$ is the complementing factor which is a perfect square.

\section{General validity of the equation $n \times z^{2}$ $\pm 1=x^{2}$}

The relationship $n \times z^{2}=t$ was discovered and developed from the concept of a cycle factor of Pythagorean triples that represent a series of triangles that approximate a rightangled triangle with one irrational side. Not all the numbers are represented in this system of cycle factors, but with the development of the algebraic relationship that links these cycle factors, could the algebraic relationship be extended to all numbers? Does the relationship where a perfect square times a number $n$ equal a number adjacent to another perfect square answer to all values of $n$, ie $x^{2}=n \times z^{2} \pm$ 1 ? Our first impulse was to systematically run through all the perfect squares, looking at the numbers 1 less, and 1 greater, factorising these numbers, looking for those that may have factors that can be described by $n \times z^{2}$. We soon realised that that would be a monstrous task, and turned it round the other way. We systematically searched for each number, starting with $n=2$ to 99 , all the solutions where $n$ $\times z^{2} \pm 1$ is a perfect square where $z$ is any number from $2-$ 300 000. The results are reported in table 4 in the form of ordered pairs in which the first number represents $\mathrm{z}$ and the second number represents $x=\sqrt{n \times z^{2} \pm 1}$. The ordered pairs which are bold are the ones that relate to cycle factors presented in table 3 . With a little consideration, it is clear that $n$ may not be a perfect square, since the product of two perfect squares is also a perfect square, and adding or subtracting 1 to a perfect square will not yield a perfect square, since for positive integers no two perfect squares are adjacent to each other. The table thus contains all the values less than 100 that are not perfect squares. The solutions where 1 is added is indicated by a + before the row, and - where 1 is subtracted.

Table 4. All the solutions for $n=2-99$ and $z=2-300000$ where $a$ perfect square is 1 more or less than a smaller perfect square times the factor $n$.

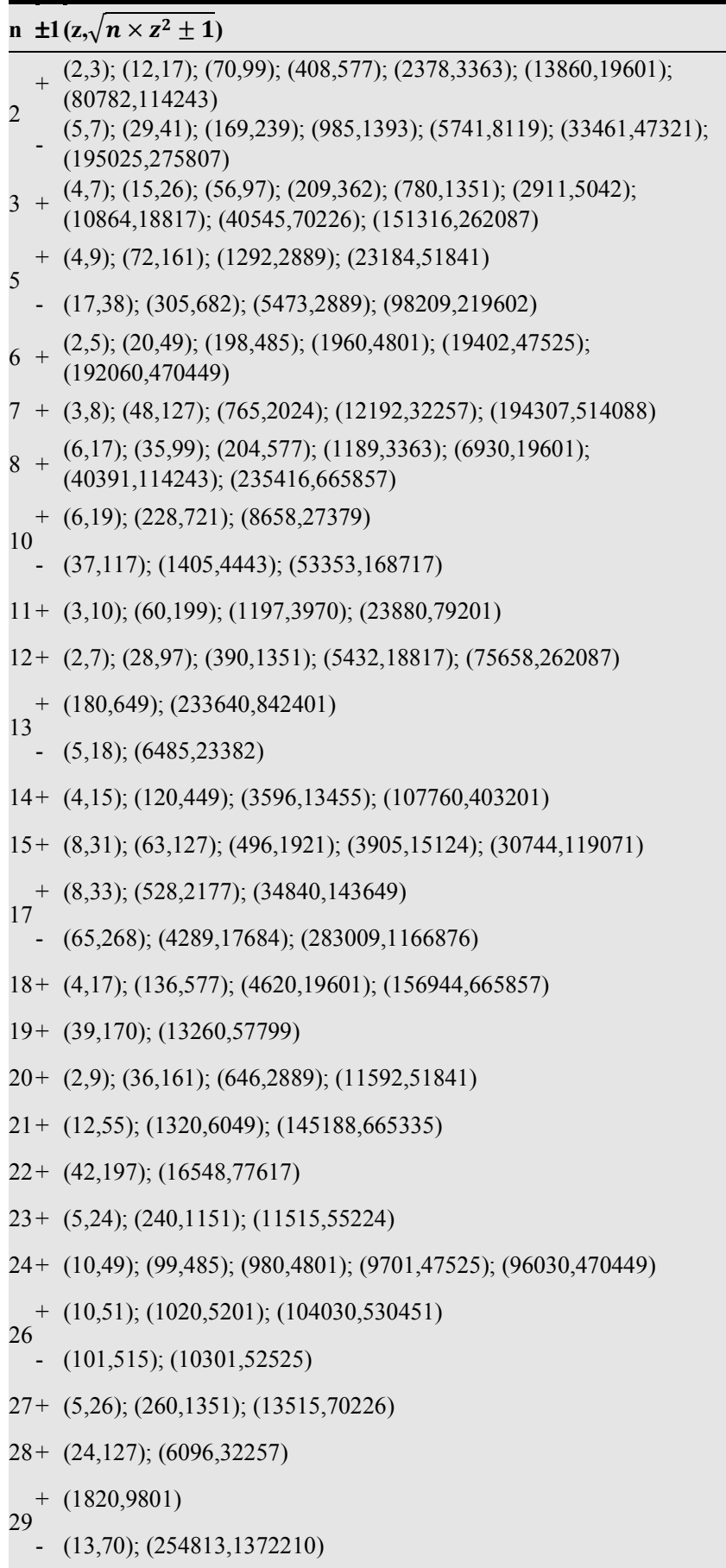




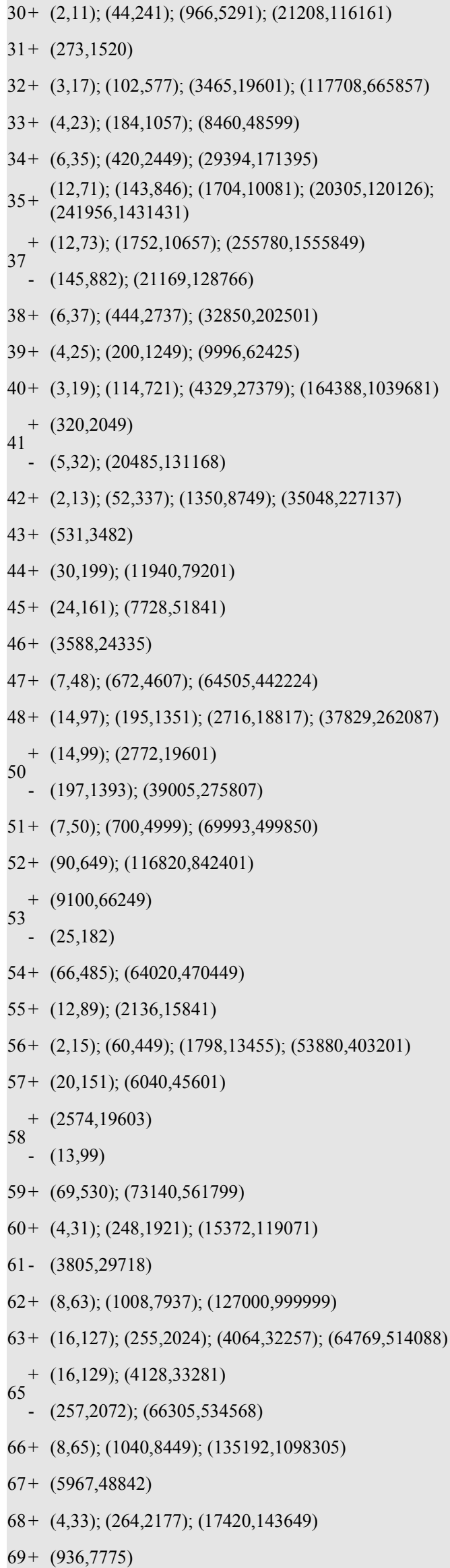

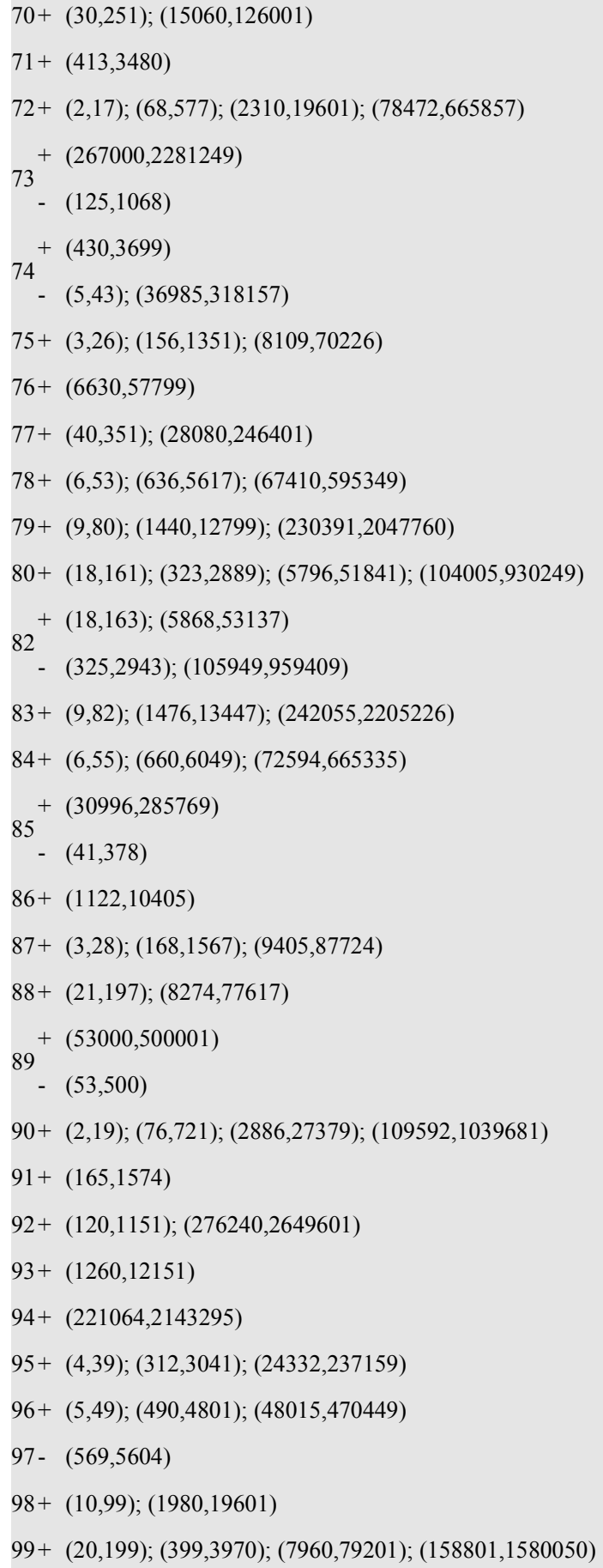

Interesting consistencies that arise in table 4 between $n$ and the calculated second number $\left.\left(\sqrt{n \times z^{2} \pm 1}\right)=x\right)$ in the ordered pair of the first solutions for $n$ are.

- $\quad$ For $n$ just below each perfect square, $x=2 n+1$, and for $n$ just above each perfect square, $x=2 n-1$.

- The first number in the solution $(z)$, both just greater than and just less than a perfect square, is two times the root of the perfect square.

- $\quad$ For the numbers $n, 2$ less than a perfect square, $x=$ $n+1$, and for numbers $n, 2$ greater than a perfect square, $x=n-1$.

- Another progression is (see diagram below). for $n=$ 
2 (2 less than the perfect square 4$), x=3$. Equidistant above the perfect square $4(n=6), x$ is 2 more, 5 . But $n=6$ is three less than perfect square $9(x=5)$, so three greater than perfect square $9(n=$ $12), x=7.12$, in turn, is four less than perfect square $16(x=7)$, so for $n=20$ (four greater than perfect square 16), $x=9$. Thus we see that for $n=$ $30, x=11$, and for $n=42, x=13$, and for $n=56, x$ $=15$, and for $n=72, x=17$, and for $n=90, x=19$.

\begin{tabular}{lllllllllllllllll}
\hline diff 2 & 2 & 3 & 3 & 4 & 4 & 5 & 5 & 6 & 6 & 7 & 7 & 8 & 8 & 9 & 9 & 10
\end{tabular}

$\begin{array}{lllllllllllllllllll}n & 2 & 4 & 6 & 9 & 12 & 16 & 20 & 25 & 30 & 36 & 42 & 49 & 56 & 64 & 72 & 81 & 90 & 100\end{array}$

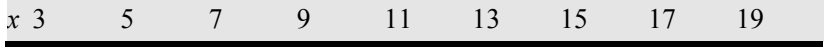

- $\quad$ For $n=$ four less than an even perfect square, $x=$ $n / 2+1$, and for $n=$ four greater than an even perfect square, $x=n / 2-1$.

- $\quad$ Beginning with perfect square 36 , for $n=\operatorname{six}$ less than a perfect square that is a multiple of $9, x=n / 3$
+1 , and for $n=\operatorname{six}$ greater than a perfect square that is a multiple of $9, x=n / 3-1$.

- $\quad$ Similarly, starting with perfect square 64 , for $n=$ eight less than a perfect square that is a multiple of $16, x=n / 4+1$, and for $n=$ eight greater than a perfect square that is a multiple of $16, x=n / 4-1$.

- $\quad$ This will probably also be true for $n=10$ less and greater than perfect squares that are multiples of 25 , starting from 100 , since for $n=90, x=90 / 5+1=$ 19.

Revisiting all the cycle factors $(y)$ of roots under 102, we find the complementing perfect square factors for them all in table 5. Note that by default even the numbers adjacent to a perfect square comply with this formula since the complementing factor is 1 , which is also a perfect square (marked in yellow). The "cycle factors" derived from table 4 without the help of a series of triangles is highlighted in red.

Table 5. Relationship of numbers adjacent to perfect squares to numbers not adjacent to perfect squares whose roots share the same cycle factors or their powers.

\begin{tabular}{|c|c|c|c|c|c|c|c|c|}
\hline Rt & Cycle factor & $n \times z^{2}$ & Rt & Cycle factor & $n \times z^{2}$ & Rt & Cycle factor & $n \times z^{2}$ \\
\hline$\sqrt{2}$ & $1+\sqrt{2}$ & $2 \times 1^{2}$ & $\sqrt{38}$ & $37+\sqrt{1368}$ & $38 \times 6^{2}$ & $\sqrt{71}$ & $3480+\sqrt{12110399}$ & $71 \times 413^{2}$ \\
\hline$\sqrt{3}$ & $2+\sqrt{3}$ & $3 \times 1^{2}$ & $\sqrt{39}$ & $25+\sqrt{624}$ & $39 \times 4^{2}$ & $\sqrt{72}$ & $17+\sqrt{288}$ & $72 \times 2^{2}$ \\
\hline$\sqrt{5}$ & $2+\sqrt{5}$ & $5 \times 1^{2}$ & $\sqrt{40}$ & $19+\sqrt{360}$ & $40 \times 3^{2}$ & $\sqrt{73}$ & $1068+\sqrt{1140625}$ & $73 \times 125^{2}$ \\
\hline$\sqrt{6}$ & $5+\sqrt{24}$ & $6 \times 2^{2}$ & $\sqrt{41}$ & $32+\sqrt{1025}$ & $41 \times 5^{2}$ & $\sqrt{74}$ & $3699+\sqrt{13682600}$ & $74 \times 430^{2}$ \\
\hline$\sqrt{7}$ & $8+\sqrt{63}$ & $7 \times 3^{2}$ & & $13+\sqrt{168}$ & $42 \times 2^{2}$ & $\sqrt{75}$ & $26+\sqrt{675}$ & $75 \times 3^{2}$ \\
\hline$\sqrt{8}$ & $3+\sqrt{8}$ & $8 \times 1^{2}$ & $\sqrt{43}$ & $3482+\sqrt{12124323}$ & $43 \times 531^{2}$ & $\sqrt{76}$ & $57799+\sqrt{334072440}$ & $76 \times 6630^{2}$ \\
\hline$\sqrt{10}$ & $3+\sqrt{10}$ & $10 \times 1^{2}$ & & $199+\sqrt{39600}$ & $44 \times 30^{2}$ & $\sqrt{77}$ & $351+\sqrt{123200}$ & $77 \times 400^{2}$ \\
\hline$\sqrt{11}$ & $10+\sqrt{99}$ & $11 \times 3^{2}$ & $\sqrt{45}$ & $161+\sqrt{25920}$ & $45 \times 24^{2}$ & & $53+\sqrt{2808}$ & $78 \times 6^{2}$ \\
\hline$\sqrt{12}$ & $7+\sqrt{48}$ & $12 \times 2^{2}$ & & $24335+\sqrt{592192224}$ & $46 \times 3588^{2}$ & $\sqrt{79}$ & $80+\sqrt{6399}$ & $79 \times 9^{2}$ \\
\hline$\sqrt{13}$ & $18+\sqrt{325}$ & $13 \times 5^{2}$ & $\sqrt{47}$ & $48+\sqrt{2303}$ & $47 \times 7^{2}$ & $\sqrt{80}$ & $9+\sqrt{80}$ & $80 \times 1^{2}$ \\
\hline$\sqrt{14}$ & $15+\sqrt{224}$ & $14 \times 4^{2}$ & $\sqrt{48}$ & $7+\sqrt{48}$ & $48 \times 1^{2}$ & $\sqrt{82}$ & $9+\sqrt{82}$ & $82 \times 1^{2}$ \\
\hline$\sqrt{15}$ & $4+\sqrt{15}$ & $15 \times 1^{2}$ & $\sqrt{50}$ & $7+\sqrt{50}$ & $50 \times 1^{2}$ & $\sqrt{83}$ & $82+\sqrt{6723}$ & $83 \times 9^{2}$ \\
\hline$\sqrt{17}$ & $4+\sqrt{17}$ & $17 \times 1^{2}$ & $\sqrt{51}$ & $50+\sqrt{2499}$ & $51 \times 7^{2}$ & & $55+\sqrt{3024}$ & $84 \times 6^{2}$ \\
\hline$\sqrt{18}$ & $17+\sqrt{288}$ & $18 \times 4^{2}$ & & $649+\sqrt{421200}$ & $52 \times 90^{2}$ & $\sqrt{85}$ & $378+\sqrt{142885}$ & $85 \times 41^{2}$ \\
\hline$\sqrt{19}$ & $170+\sqrt{28899}$ & $19 \times 39^{2}$ & $\sqrt{53}$ & $182+\sqrt{33125}$ & $53 \times 25^{2}$ & & $10405+\sqrt{108264024}$ & $86 \times 1122^{2}$ \\
\hline$\sqrt{20}$ & $9+\sqrt{80}$ & $20 \times 2^{2}$ & & $485+\sqrt{235224}$ & $54 \times 66^{2}$ & $\sqrt{87}$ & $28+\sqrt{783}$ & $87 \times 3^{2}$ \\
\hline$\sqrt{21}$ & $55+\sqrt{3024}$ & $21 \times 12^{2}$ & $\sqrt{55}$ & $89+\sqrt{7920}$ & $55 \times 12^{2}$ & $\sqrt{88}$ & $197+\sqrt{38808}$ & $88 \times 21^{2}$ \\
\hline$\sqrt{22}$ & $197+\sqrt{38808}$ & $22 \times 42^{2}$ & $\sqrt{56}$ & $15+\sqrt{224}$ & $56 \times 2^{2}$ & $\sqrt{89}$ & $500+\sqrt{250001}$ & $89 \times 53^{2}$ \\
\hline$\sqrt{23}$ & $24+\sqrt{575}$ & $23 \times 5^{2}$ & $\sqrt{57}$ & $151+\sqrt{22800}$ & $57 \times 20^{2}$ & & $19+\sqrt{360}$ & $90 \times 2^{2}$ \\
\hline$\sqrt{24}$ & $5+\sqrt{24}$ & $24 \times 1^{2}$ & $\sqrt{58}$ & $19603+\sqrt{384277608}$ & $58 \times 2574^{2}$ & $\sqrt{91}$ & $1574+\sqrt{2477475}$ & $91 \times 165^{2}$ \\
\hline$\sqrt{26}$ & $5+\sqrt{26}$ & $26 \times 1^{2}$ & $\sqrt{59}$ & $530+\sqrt{280899}$ & $59 \times 69^{2}$ & $\sqrt{92}$ & $1151+\sqrt{1324800}$ & $92 \times 1260^{2}$ \\
\hline$\sqrt{27}$ & $26+\sqrt{675}$ & $27 \times 5^{2}$ & & $31+\sqrt{960}$ & $60 \times 4^{2}$ & $\sqrt{93}$ & $12151+\sqrt{147646800}$ & $93 \times 1260^{2}$ \\
\hline$\sqrt{28}$ & $127+\sqrt{16128}$ & $28 \times 24^{2}$ & $\sqrt{61}$ & $29718+\sqrt{883159525}$ & $61 \times 3805^{2}$ & & $2143295+$ & $24 \times 24064^{2}$ \\
\hline$\sqrt{29}$ & $70+\sqrt{4901}$ & $29 \times 13^{2}$ & & $63+\sqrt{3968}$ & $62 \times 8^{2}$ & & $\sqrt{4593713457}$ & $94 \times 221064^{2}$ \\
\hline$\sqrt{30}$ & $11+\sqrt{120}$ & $30 \times 2^{2}$ & $\sqrt{63}$ & $8+\sqrt{63}$ & $63 \times 1^{2}$ & $\sqrt{95}$ & $39+\sqrt{1520}$ & $95 \times 4^{2}$ \\
\hline$\sqrt{31}$ & $1520+\sqrt{2310399}$ & $31 \times 273^{2}$ & $\sqrt{65}$ & $8+\sqrt{65}$ & $65 \times 1^{2}$ & $\sqrt{96}$ & $49+\sqrt{2400}$ & $96 \times 5^{2}$ \\
\hline$\sqrt{32}$ & $17+\sqrt{288}$ & $32 \times 3^{2}$ & & $65+\sqrt{4224}$ & $66 \times 8^{2}$ & $\sqrt{97}$ & $5604+\sqrt{31404817}$ & $97 \times 569^{2}$ \\
\hline$\sqrt{33}$ & $23+\sqrt{528}$ & $33 \times 4^{2}$ & $\sqrt{67}$ & $48842+\sqrt{2385540963}$ & $67 \times 5967^{2}$ & $\sqrt{98}$ & $99+\sqrt{9800}$ & $98 \times 10^{2}$ \\
\hline$\sqrt{34}$ & $35+\sqrt{1224}$ & $34 \times 6^{2}$ & & $33+\sqrt{1088}$ & $68 \times 4^{2}$ & $\sqrt{99}$ & $10+\sqrt{99}$ & $99 \times 1^{2}$ \\
\hline$\sqrt{35}$ & $6+\sqrt{35}$ & $35 \times 1^{2}$ & $\sqrt{69}$ & $7775+\sqrt{60450624}$ & $69 \times 936^{2}$ & $\sqrt{101}$ & $10+\sqrt{101}$ & $101 \times 1^{2}$ \\
\hline$\sqrt{37}$ & $6+\sqrt{37}$ & $37 \times 1^{2}$ & $\sqrt{ } 70$ & $251+\sqrt{63000}$ & $70 \times 30^{2}$ & & & \\
\hline
\end{tabular}

- An interesting observation is that the roots of the numbers that are 2 greater or less than a perfect square (excepting for 2 ) have as their $z^{2}$ factors the perfect square. For example, $79 \times 9^{2}$ and $83 \times 9^{2}$ respectively less than and greater than $9^{2}=81$.

- All values for $n$ below 102 have solutions, even though some, such as $\sqrt{94}$, had to go a very long way before a solution was found. 


\section{Powers of Cycle Factors $\left(y^{m}\right)$}

It was then found that raising the cycle factors in their powers (Table 6) produced interesting results. In the table, where a number exceeds $10^{13}$, the cells are left blank. On the left of the table, the square roots whose cycle factors begin with a number just greater than an integer are highlighted in yellow (The rest begin with cycle factors that are just less than an integer.). The square roots whose cycle factors were not derived from series of triangles are highlighted in green. All the powers of cycle factors whose decimals when rounded off do not show whether the number is greater than or less than an integer are highlighted in yellow. All the other highlighting colours associate cycle factors or their powers with those of other square roots. Green, for instance at $\sqrt{2}$ shows powers of cycle factors that correlate with cycle factors at $\sqrt{8}$, and many other square roots in the table.

Table 6. Powers of the cycle factors.

\begin{tabular}{|c|c|c|c|c|c|c|c|}
\hline Rt & Cycle factor (y) & $y^{2}$ & $y^{3}$ & $y^{4}$ & $y^{5}$ & $y^{6}$ & $y^{7}$ \\
\hline$\sqrt{2}$ & 2.41421356237 & 5.82842712475 & 14.0710678119 & 33.9705627485 & 82.0121933088 & 197.994949366 & 478.002092041 \\
\hline$\sqrt{3}$ & 3.73205080757 & 13.9282032303 & 51.9807621135 & 193.994845224 & 723.998618782 & 2701.99962990 & 10083.9999008 \\
\hline$\sqrt{5}$ & 4.23606797750 & 17.9442719100 & 76.0131556175 & 321.996894380 & 1364.00073314 & 5777.99982693 & 24476.0000409 \\
\hline$\sqrt{6}$ & 9.89897948557 & 97.9897948557 & 969.998969071 & 9601.99989586 & 95049.9999895 & 940897.999999 & 9313930.00000 \\
\hline$\sqrt{7}$ & 15.9372539332 & 253.996062931 & 4047.99975296 & 64513.9999845 & 1028176.00000 & 16386302.0000 & 261152656.000 \\
\hline$\sqrt{8}$ & 5.82842712475 & 33.9705627485 & 197.994949366 & 1153.99913345 & 6725.99985132 & 39201.9999745 & 228485.999996 \\
\hline$\sqrt{10}$ & 6.16227766017 & 37.9736659610 & 234.004273426 & 1441.99930652 & 8886.00011254 & 54757.9999817 & 337434.000003 \\
\hline$\sqrt{11}$ & 19.9498743711 & 397.997487421 & 7939.99987406 & 158401.999994 & 3160100.00000 & 63043598.0000 & 1257711860.00 \\
\hline$\sqrt{12}$ & 13.9282032303 & 193.994845224 & 2701.99962990 & 37633.9999734 & 524173.999998 & 7300802.00000 & 101687054.000 \\
\hline$\sqrt{13}$ & 36.0277563773 & 1297.99922958 & 46764.0000214 & 1684802.00000 & 60699636.0000 & 2186871698.00 & 78788080764.0 \\
\hline$\sqrt{14}$ & 29.9666295471 & 897.998886413 & 26909.9999628 & 806401.999999 & 24165150.0000 & 724148098.000 & 21700277790.0 \\
\hline$\sqrt{15}$ & 7.87298334621 & 61.9838667697 & 487.997950811 & 3841.99973972 & 30247.9999669 & 238141.999996 & 1874888.00000 \\
\hline$\sqrt{17}$ & 8.12310562562 & 65.9848450049 & 536.001865665 & 4353.99977033 & 35368.0000283 & 287297.999997 & 2333752.00000 \\
\hline$\sqrt{18}$ & 33.9705627485 & 1153.99913345 & 39201.9999745 & 1331714.00000 & 45239074.0000 & 1536796802.00 & 52205852194.0 \\
\hline$\sqrt{19}$ & 339.997058798 & 115597.999991 & 39302980.0000 & 13362897602.0 & & & \\
\hline$\sqrt{20}$ & 17.9442719100 & 321.996894380 & 5777.99982693 & 103681.999990 & 1860498.00000 & 33385282.0000 & 599074578.000 \\
\hline$\sqrt{21}$ & 109.990908339 & 12097.9999173 & 1330670.00000 & 146361602.000 & 16098445550.0 & & \\
\hline$\sqrt{22}$ & 393.997461913 & 155233.999994 & 61161802.0000 & 24097594754.0 & & & \\
\hline$\sqrt{23}$ & 47.9791576166 & 2301.99956560 & 110447.999991 & 5299202.00000 & 254251248.000 & 12198760702.0 & 585286262448 \\
\hline$\sqrt{24}$ & 9.89897948557 & 97.9897948557 & 969.998969071 & 9601.99989586 & 95049.9999895 & 940897.999999 & 9313930.00000 \\
\hline$\sqrt{26}$ & 10.0990195136 & 101.990195136 & 1030.00097087 & 10401.9999039 & 105050.000010 & 1060902.00000 & 10714070.0000 \\
\hline$\sqrt{27}$ & 51.9807621135 & 2701.99962990 & 140451.999993 & 7300802.00000 & 379501252.000 & 19726764302.0 & \\
\hline$\sqrt{28}$ & 253.996062931 & 64513.9999845 & 16386302.0000 & 4162056194.00 & & & \\
\hline$\sqrt{29}$ & 140.007142493 & 19601.9999490 & 2744420.00000 & 384238402.000 & 53796120700.0 & & \\
\hline$\sqrt{30}$ & 21.9544511501 & 481.997925302 & 10581.9999055 & 232321.999996 & 5100502.00000 & 111978722.000 & 2458431382.00 \\
\hline$\sqrt{31}$ & 3039.99967105 & 9241598.00000 & 28094454880.0 & & & & \\
\hline$\sqrt{32}$ & 33.9705627485 & 1153.99913345 & 39201.9999745 & 1331714.00000 & 45239074.0000 & 1536796802.00 & 52205852194.0 \\
\hline$\sqrt{33}$ & 45.9782505862 & 2113.99952696 & 97197.9999897 & 4468994.00000 & 205476526.000 & 9447451202.00 & 434377278766 \\
\hline$\sqrt{34}$ & 69.9857113691 & 4897.99979584 & 342789.999997 & 23990402.0000 & 1678985350.00 & 117504984098 . & \\
\hline$\sqrt{35}$ & 11.9160797831 & 141.992957397 & 1691.99940898 & 20161.9999504 & 240251.999996 & 2862862.00000 & 34114092.0000 \\
\hline$\sqrt{37}$ & 12.0827625303 & 145.993150364 & 1764.00056689 & 21313.9999531 & 257532.000004 & 3111698.00000 & 37597908.0000 \\
\hline$\sqrt{38}$ & 73.9864840178 & 5473.99981732 & 405001.999998 & 29964674.0000 & 2216980874.00 & 164026620002. & \\
\hline$\sqrt{39}$ & 49.9799919936 & 2497.99959968 & 124849.999992 & 6240002.00000 & 311875250.000 & 15587522498.0 & 779064249650 \\
\hline$\sqrt{40}$ & 37.9736659610 & 1441.99930652 & 54757.9999817 & 2079362.00000 & 78960998.0000 & 2998438562.00 & 113861704358 \\
\hline$\sqrt{41}$ & 64.0156211872 & 4097.99975598 & 262336.000004 & 16793602.0000 & 1075052864.00 & 68820176898.0 & \\
\hline$\sqrt{42}$ & 25.9614813968 & 673.998516317 & 17497.9999429 & 454273.999998 & 11793626.0000 & 306180002.000 & 7948886426.00 \\
\hline$\sqrt{43}$ & 6963.99985640 & 48497294.0000 & 337735148452 . & & & & \\
\hline$\sqrt{44}$ & 397.997487421 & 158401.999994 & 63043598.0000 & 25091193602.0 & & & \\
\hline
\end{tabular}


Martin W Bredenkamp: Geometric Series of Numbers Approximating Positive Integers

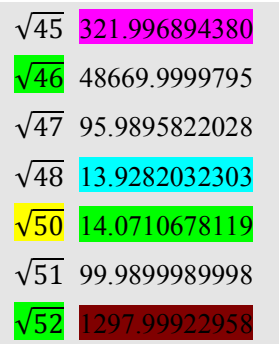

$\sqrt{53} 364.002747232$

$\sqrt{54} 969.998969071$

$\sqrt{55} \quad 177.994381845$

$\sqrt{56} 29.9666295471$

$\sqrt{57} 301.996688705$

$\sqrt{58} \quad 39205.9999745$

$\begin{array}{ll}\sqrt{59} & 1059.99905660\end{array}$

$\sqrt{60} \quad 61.9838667697$

$\begin{array}{lll}\sqrt{61} & 59436.0000168\end{array}$

$\begin{array}{lll}\sqrt{62} & 125.992062992\end{array}$

$\begin{array}{lll}\sqrt{63} & 15.9372539332\end{array}$

$\begin{array}{lll}\sqrt{65} & 16.0622577483\end{array}$

$\begin{array}{ll}\sqrt{66} & 129.992307237\end{array}$

$\begin{array}{ll}\sqrt{67} & 97683.9999898\end{array}$

$\sqrt{68} 65.9848450049$

$\begin{array}{lll}\sqrt{69} & 15549.9999357\end{array}$

$\sqrt{70} 501.998007960$

$\begin{array}{ll}\sqrt{71} & 6959.99985632\end{array}$

$\begin{array}{lll}\sqrt{72} & 33.9705627485\end{array}$

$\begin{array}{ll}\sqrt{73} & 2136.00046816\end{array}$

$\sqrt{74} 7397.99986483$

$\sqrt{75} 51.9807621135$

$\sqrt{76} 115597.999991$

$\sqrt{77} \quad 701.998575496$

$\begin{array}{lll}\sqrt{78} & 105.990565198\end{array}$

$\begin{array}{lll}\sqrt{79} & 159.993749756\end{array}$

$\sqrt{80} \quad 17.9442719100$

$\begin{array}{lll}\sqrt{82} & 18.0553851381\end{array}$

$\begin{array}{ll}\sqrt{83} & 163.993902212\end{array}$

$\sqrt{84} 109.990908339$

$\begin{array}{lll}\sqrt{85} & 756.001322749\end{array}$

$\sqrt{\sqrt{86}} 20809.9999519$

$\sqrt{87} 55.9821371593$

$\begin{array}{lll}\sqrt{88} & 393.997461913\end{array}$

$\begin{array}{lll}\sqrt{89} & 1000.00100000\end{array}$

$\sqrt{90} \quad 37.9736659610$ $\sqrt{91} 3147.99968234$

$\sqrt{92} 2301.99956560$

$\sqrt{93} 24301.9999589$

$\sqrt{94} 4286590.00000$

$\sqrt{95} 77.9871773792$

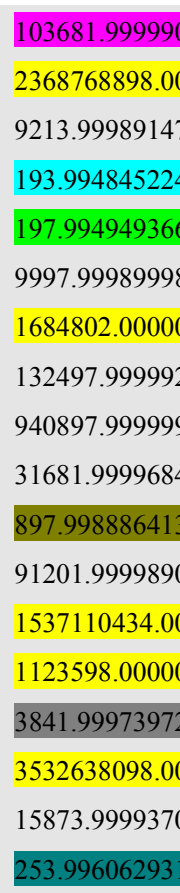

257.996123973

16897.9999408

9542163854.00

4353.99977033

241802498.000

252001.999996

48441598.0000

1153.99913345

4562498.00000

54730402.0000

2701.99962990

13362897602.0

492801.999998

11233.9999110

25597.9999609

321.996894380

325.996932486

26893.9999628

12097.9999173

571537.999998

433056098.000

3133.99968092

155233.999994

1000002.00000

1441.99930652

9909902.00000

5299202.00000

590587202.000

6081.99983558
33385282.0000

10749957122.0

$884447.999999 \quad 84897794.0000$

2701.99962990

2786.00035894

999699.999999

2186871698.00

48229636.0000

912670090.000

5639218.00000

26909.9999628

27542702.0000

37633.9999734

39201.9999745

99960002.0000

17555720002.0

885289046402 .

1003749122.00

806401.999999

8317804802.00

1191012820.00

238141.999996

1999998.00000

4047.99975296

4144.00024131

2196610.00000

287297.999997

126504502.000

337153515120 .

39201.9999745

9745497864.00

404895506598.

140451.999993

345946302.000

1190698.00000

4095520.00000

5777.99982693

5886.00016989

4410452.00000

1330670.00000

432083484.000

175447.999994

61161802.0000

1000003000.00

54757.9999817

31196368348.0

12198760702.0

251983874.000

64513.9999845

66561.9999850

285542402.000

18957314.0000

63505008002.0

1331714.00000

7300802.00000

242853811202.

126202754.000

655257602.000

103681.999990

106273.999991

723287234.000

146361602.000

326655685442 .

9821954.00000

24097594754.0

2079362.00000
178661704498.

24165150.0000

45239074.0000

1536796802.00

52205852194.0

549853976.000

782248264702. 7300802.00000 7761798.00000 999400089998.

724148098.000

31747968126.0

1069136.00000

16386302.0000

17172738.0000

37118315650.0

1250895426.00

82540140802.0

379501252.000

19726764302.0

13376301226.0

104837120800.

1860498.00000

1918818.00000

33385282.0000

599074578.000

34644998.0000

625528782.000

118614695924.

16098445550.0

78960998.0000

2998438562.00

113861704358.
1028176.00000 21700277790.0

261152656.000

275832944.000

101687054.000 109216786.000

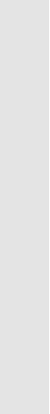




\begin{tabular}{llllllll}
$\sqrt{96}$ & 97.9897948557 & 9601.99989586 & 940897.999999 & 92198402.0000 & 9034502498.00 & 885289046402. \\
$\sqrt{97}$ & 11208.0000892 & 125619266.000 & & & & & \\
$\sqrt{98}$ & 197.994949366 & 39201.9999745 & 7761798.00000 & 1536796802.00 & 304278004998. & & \\
$\sqrt{99}$ & 19.9498743711 & 397.997487421 & 7939.99987406 & 158401.999994 & 3160100.00000 & 63043598.0000 & 1257711860.00 \\
$\sqrt{101} 20.0498756211$ & 401.997512422 & 8060.00012407 & 161601.999994 & 3240100.00000 & 64963602.0000 & 1302512140.00 \\
\hline
\end{tabular}

The following observations may be made with respect to raising cycle factors to their powers.

- $\quad$ Powers of the cycle factors come closer to positive integers as they increase.

- The rule that applies to the cycle factors that are not adjacent to a perfect square $\left(n \times z^{2}=t\right)$ also applies to the powers of a cycle factors. Eg the cycle factor of $\sqrt{7}(8+\sqrt{63})$ is the same as for the cycle factor of $\sqrt{7 \times 3^{2}}=\sqrt{63}$. Likewise the square of the cycle factor for these numbers $(127+\sqrt{16128}=$ $253.996062931)$ is also the cycle factor of $\sqrt{16128}$. But $16128=2^{8} \cdot 3^{2} \cdot 7$, therefore $16128=4032 \times 2^{2}=$ $1792 \times 3^{2}=1008 \times 4^{2}=448 \times 6^{2}=252 \times 8^{2}=112$ $\times 12^{2}=63 \times 16^{2}=7 \times 48^{2}$. Therefore the square roots of $7,63,112,252,448,1008,1792,4032$ and 16128 share the same cycle factors and/or powers of cycle factors. A limited amount of this can be seen in table 6 (all colour coded with colours other than yellow).

- Consider all the bright green highlighted cycle factors and powers of cycle factors. $\sqrt{2}$ shares in the cycle factors and powers of cycle factors with $\sqrt{2 \times 2^{2}}=\sqrt{8}, \sqrt{2 \times 3^{2}}=\sqrt{18}, \sqrt{2 \times 4^{2}}=\sqrt{32}$, $\sqrt{2 \times 5^{2}}=\sqrt{50}, \sqrt{2 \times 6^{2}}=\sqrt{72}$ and $\sqrt{2 \times 7^{2}}=$ $\sqrt{98}$. Likewise each other colour also relates different cycle factors and their powers (Binary colours were used when we ran out of colours).

- Looking at the multiple solutions for the ordered pairs in table $4\left(z, \sqrt{n \times z^{2} \pm 1}\right)$, we find that the respective solutions represent the respective powers of the cycle factors for each number $n$. Eg for $n=7$, the first solution is for $z=3$ and $x=8$. This means that the cycle factor is the same as for the square root of $7 \times 3^{2}=63$, which is just short of $8^{2}$. The cycle factor for $\sqrt{63}$ is $8+\sqrt{63}$. As shown earlier, $(8+\sqrt{63})^{2}=127+\sqrt{16128}=253.996062931$. $\sqrt{16128 / 7}=48$. 16128 is $127^{2}-1$, giving an ordered pair $(48,127)$, the second solution to $n=7$ in table 4 , etc. Note that when working with $n$ just greater or less than a perfect square, the solution in table 4 starts with the first power of the cycle factor and not the cycle factor itself as for the square roots of numbers not adjacent to a perfect square.

Taking a further look at table 4 and its solutions to cycle factors and their powers, the following is apparent.

- Only for $n=61$ and 97 there seem to be no addition solutions (solutions where 1 must be added to $n \times z^{2}$ ) below 300 000. Several numbers have addition and subtraction solutions. When they do, the values alternate, therefore, values of $n$ that have more than one addition solution do not have any subtraction solutions even beyond 300 000. Only the numbers that directly follow perfect squares alternate beginning with an addition solution. All the other cases that have addition and subtraction solutions begin with a subtraction solution first. It turns out that 61 and 97 belong to that category except that their respective next solutions, which are addition solutions, are greater than 300000 .

- 4 greater than every uneven perfect square has subtraction and addition solutions beginning with subtraction, in contrast with the numbers that follow a perfect square which begin with an addition solution.

- All the numbers that have addition and subtraction solutions are the sum of two perfect squares. Not all the sums of two perfect squares, however, have both addition and subtraction solutions.

- $\quad$ Table 7 shows a matrix of all the numbers up to 100 that can be made up of the sum of two perfect squares, and whether they have addition and then subtraction (+ superscript), or subtraction and then addition (- superscript) solutions, as well as those that are in themselves perfect squares (* superscript) and thus not have any solutions. Those that are addition solutions only have no superscript. All the numbers below the stepped double line are a repetition of information above the stepped double line.

Table 7. A matrix that indicates all the sums of two perfect squares less than or equal to 100 showing their relation to the formula $n+z^{2} \pm 1=x^{2}$.

\begin{tabular}{|c|c|c|c|c|c|c|c|c|c|}
\hline & 1 & 4 & 9 & 16 & 25 & 36 & 49 & 64 & 81 \\
\hline 1 & $2^{+}$ & $5^{+}$ & $10^{+}$ & $17^{+}$ & $26^{+}$ & $37^{+}$ & $50^{+}$ & $65^{+}$ & $82^{+}$ \\
\hline 4 & $5^{+}$ & 8 & $13^{-}$ & 20 & $29^{-}$ & 40 & $53^{-}$ & 68 & $85^{\circ}$ \\
\hline 9 & $10^{+}$ & $13^{-}$ & 18 & $25^{*}$ & 34 & 45 & $58^{-}$ & $73^{-}$ & 90 \\
\hline 16 & $17^{+}$ & 20 & $25^{*}$ & 32 & $41^{-}$ & 52 & $65^{-}$ & 80 & $97^{\circ}$ \\
\hline 25 & $26^{+}$ & $29^{-}$ & 34 & $41^{-}$ & $50^{+}$ & $61^{-}$ & $74^{-}$ & $89^{-}$ & \\
\hline 36 & $37^{+}$ & 40 & 45 & 52 & $61^{-}$ & 72 & $85^{-}$ & $100^{*}$ & \\
\hline 49 & $50^{+}$ & $53^{-}$ & $58^{-}$ & $65^{-}$ & $74^{-}$ & $85^{-}$ & 98 & & \\
\hline 64 & $65^{+}$ & 68 & $73^{-}$ & 80 & $89^{-}$ & $100^{*}$ & & & \\
\hline 81 & $82^{+}$ & $85^{-}$ & 90 & $97^{-}$ & & & & & \\
\hline
\end{tabular}


Patterns are visible for the first two rows/columns. The fourth row/column also has all the uneven sums that include 16 as addition and subtraction solutions, except 25 because it is a perfect square in itself. All the uneven numbered sums throughout seem to be a part of the addition and subtraction solution system except $45(36+9)$, but besides these, there is no apparent pattern. This may become clear if the matrix is expanded. $2 \times 25$ is the only number away from the first row/column that is positive, because it duplicates $1+49$ in the first column. The diagonal does not have addition and subtraction solutions, other than the first row/column and 50.

All these patterns provide a clue as to how these systems work and need further investigation.

\section{Prime factorizing the root of the perfect square factor $(\mathrm{z})$}

Taking another look at table 6 , and applying the formula $n \times z^{2} \pm 1=x^{2}$ it turns out that for each power of a cycle factor, $n$ is a factor of the square of the integer portion $(x)$ of any power of the cycle factor plus or minus 1, and the remaining factor is a perfect square $\left(z^{2}\right)$.

- The first observation that needs to be made is that for each power of the cycle factor, the integer portion $(x)$ is like the integer portion of the cycle factor itself, and is equal to the $x$-component of the corresponding solution in table 4 . For example. the integer portion of the powers of the cycle factor of 7 are in increasing order (the closest integer to half the cycle factor or its power). $8,127,2024,32257$, $514088, \ldots$ (table 6 ). The $x$-part of the solutions for 7 in table 4 are. $8,127,2024,32257,514088$. When applying the formula $z=\sqrt{\left(x^{2} \pm 1\right) / n}$ [where $x$ is the integer portion of the cycle factor or its power, approximately half of the cycle factor $(y)$ or power $\left(y^{m}\right)$ ] the $z$-part of the solution in table 4 is produced. $3,48,765,12192,194307, \ldots$.

- Because the calculated part $\left(z=\sqrt{\left(x^{2} \pm 1\right) / n}\right)$ renders a perfect-square factor, the root of the perfect square $(z)$ is prime factorised for investigation (table 8). Very clear patterns are visible as the powers increase. Using the cycle factor of the $\sqrt{7}$ as an example to illustrate the patterns, we see the following.

- Some factors appear in the solutions of every power (3), some in every second power (24), some every third power $(5,17$ and a second 3$)$, some in every fourth power (127 and a fifth 2), some in every fifth power (239 and 271), some in every sixth power (11 and 23), etc.

- 2 behaves binary in that it occurs at least 4 times in every second power, at least 5 times in every fourth power, at least 6 times in every eighth power etc.

- 3 behaves ternary in that it occurs in every power, at least 2 times in every third power, and at least 3 times in every ninth power.

$\circ$ In the power series of the cycle factor of $\sqrt{11}$, 5 occurs every second power, and at least twice in every tenth power, showing it is quinternary.

Table 8. The prime factors of the roots of the perfect-square factors after processing the powers of cycle factors like the cycle factors themselves were processed.

\begin{tabular}{|c|c|c|c|c|c|c|c|c|c|c|}
\hline$n$ & power & $x^{2}$ & & $x^{3}$ & $x^{5}$ & $x^{6}$ & $x^{7}$ & $x^{9}$ & $x^{10}$ & $x^{11}$ \\
\hline \multirow{5}{*}{2} & $z$ & 2 & & 5 & 29 & 70 & 169 & 985 & 2378 & 5741 \\
\hline & factorised & 2 & & 5 & $2^{2} \cdot 3$ & $2 \cdot 5 \cdot 7$ & $2^{3} \cdot 3 \cdot 17$ & $5 \cdot 197$ & $2 \cdot 29 \cdot 41$ & 5741 \\
\hline & power & $x^{12}$ & & $x^{13}$ & $x^{14}$ & $x^{15}$ & $x^{16}$ & $x^{17}$ & \multicolumn{2}{|r|}{$x^{18}$} \\
\hline & $z$ & 13860 & & 33461 & 80782 & 195025 & 470832 & 1136689 & \multicolumn{2}{|r|}{2744210} \\
\hline & factorised & $2^{2} \cdot 3^{2} \cdot 5 \cdot 7 \cdot 11$ & & 33461 & $2 \cdot 13^{2} \cdot 239$ & $5^{2} \cdot 29 \cdot 269$ & $2^{4} \cdot 3 \cdot 17 \cdot 577$ & $137 \cdot 8297$ & & $2 \cdot 5 \cdot 7 \cdot 197 \cdot 199$ \\
\hline \multirow{6}{*}{3} & power & $x$ & $x^{2}$ & $x^{3}$ & $x^{4}$ & $x^{5}$ & $x^{6}$ & $x^{7}$ & $x^{8}$ & $x^{9}$ \\
\hline & $z$ & 1 & 4 & 15 & 56 & 209 & 780 & 2911 & 10864 & 40545 \\
\hline & factorised & - & $2^{2}$ & $3 \cdot 5$ & $2^{3} \cdot 7$ & $11 \cdot 19$ & $2^{2} \cdot 3 \cdot 5 \cdot 13$ & $41 \cdot 71$ & $2^{4} \cdot 7 \cdot 97$ & $3^{2} \cdot 5 \cdot 17 \cdot 53$ \\
\hline & power & $x^{10}$ & \multicolumn{2}{|r|}{$x^{11}$} & \multicolumn{2}{|c|}{$x^{12}$} & $x^{13}$ & \multicolumn{2}{|l|}{$x^{14}$} & $x^{15}$ \\
\hline & $z$ & \multicolumn{2}{|l|}{151316} & 564719 & \multicolumn{2}{|c|}{2107560} & 7865521 & \multicolumn{2}{|l|}{29354524} & 109552575 \\
\hline & factorised & \multicolumn{2}{|l|}{$2^{2} \cdot 11 \cdot 19 \cdot 181$} & $23 \cdot 43 \cdot 571$ & \multicolumn{2}{|c|}{$2^{3} \cdot 3 \cdot 5 \cdot 7 \cdot 13 \cdot 193$} & $2131 \cdot 3691$ & \multicolumn{2}{|c|}{$2^{2} \cdot 41 \cdot 71 \cdot 2521$} & $\cdot 5^{2} \cdot 11 \cdot 19 \cdot 29 \cdot 241$ \\
\hline \multirow{3}{*}{5} & power & $x$ & $x^{2}$ & $x^{3}$ & $x^{4}$ & $x^{5}$ & $x^{6}$ & $x^{7}$ & $x^{8}$ & $x^{9}$ \\
\hline & $z$ & 1 & 4 & 17 & 72 & 305 & 1292 & 5473 & 23184 & 98209 \\
\hline & factorised & - & $2^{2}$ & 17 & $2^{3} \cdot 3^{2}$ & $5 \cdot 61$ & $2^{2} \cdot 17 \cdot 19$ & $13 \cdot 421$ & $2^{4} \cdot 3^{2} \cdot 7 \cdot 23$ & $17 \cdot 53 \cdot 109$ \\
\hline
\end{tabular}




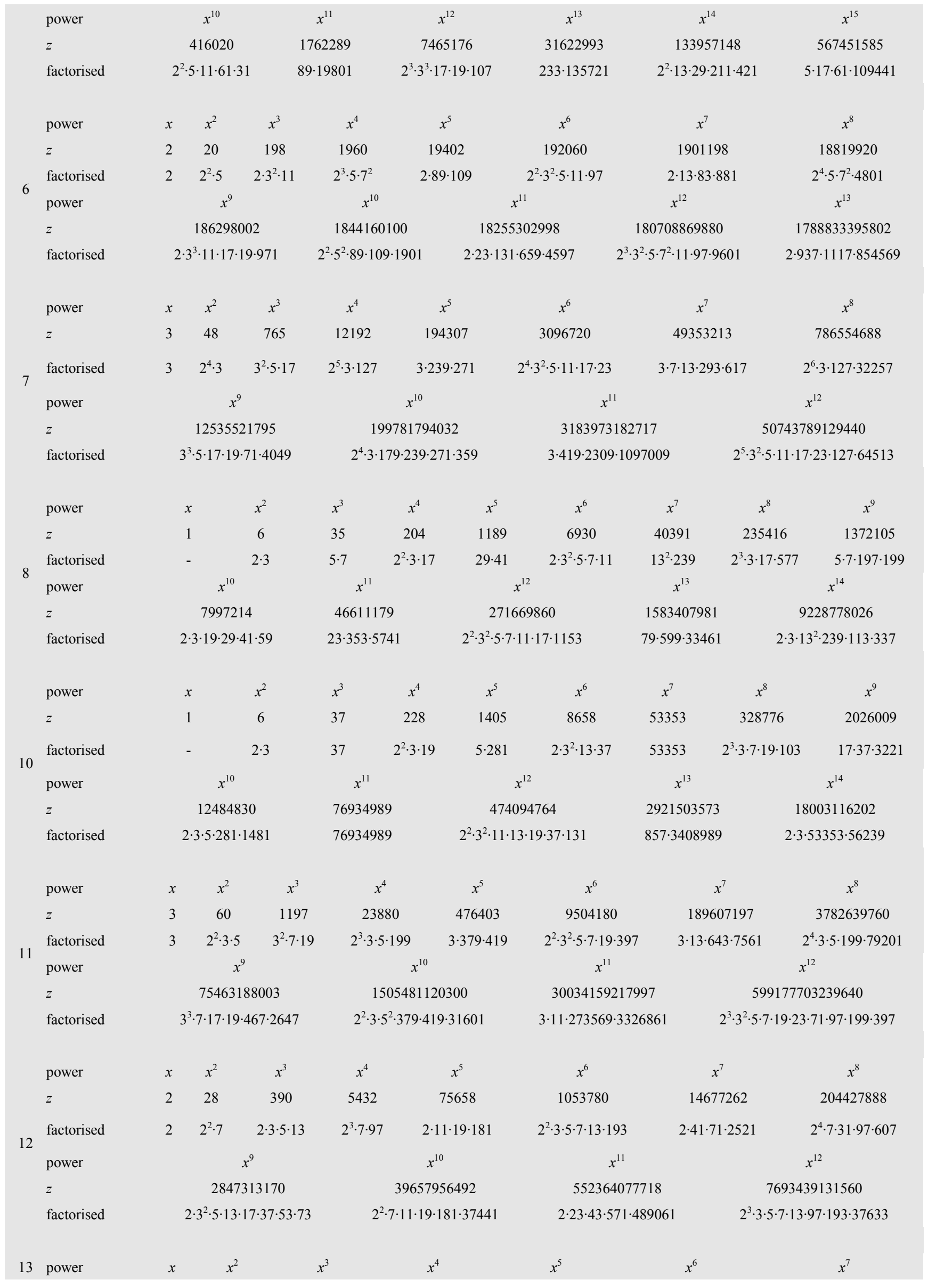




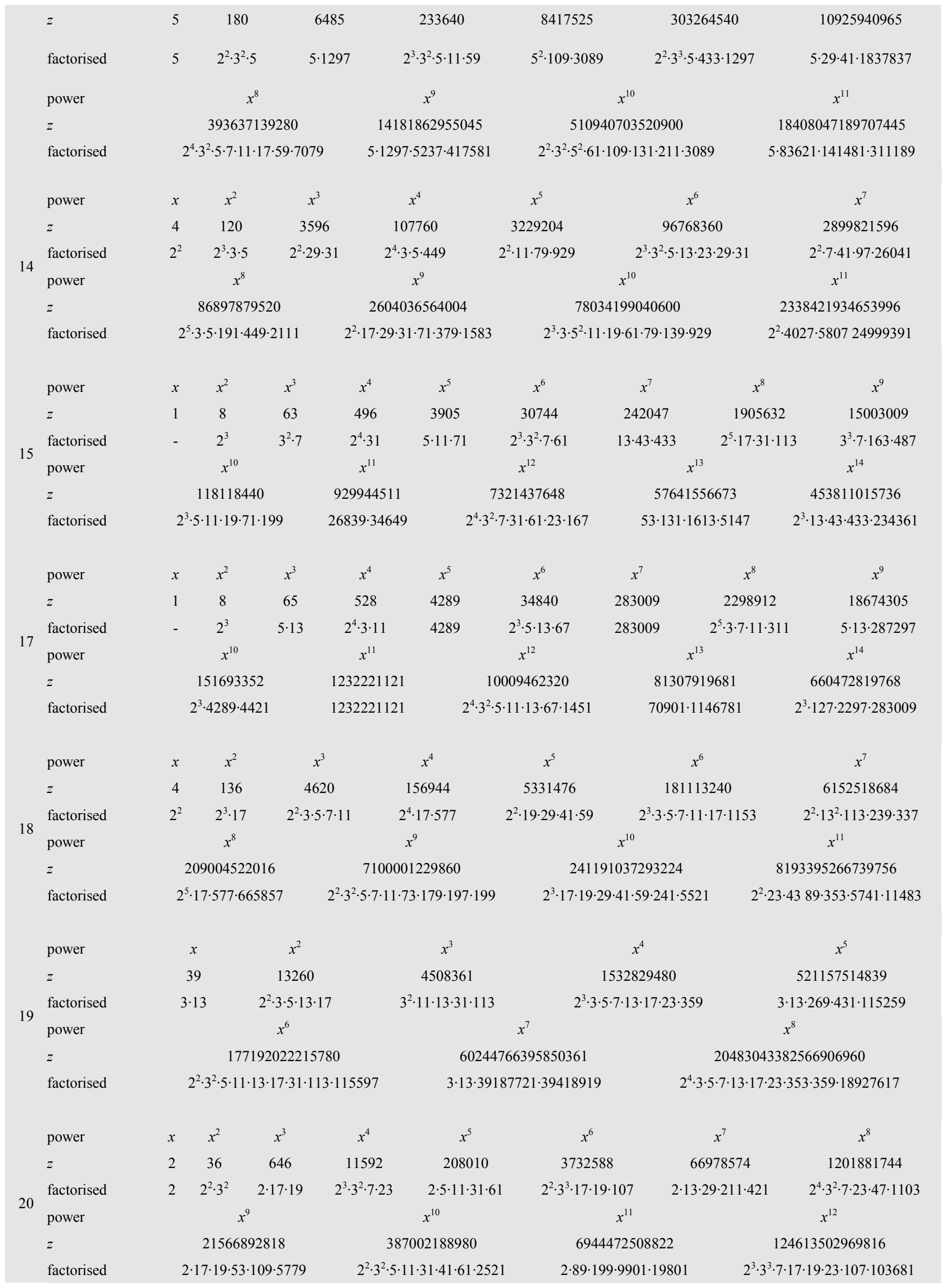




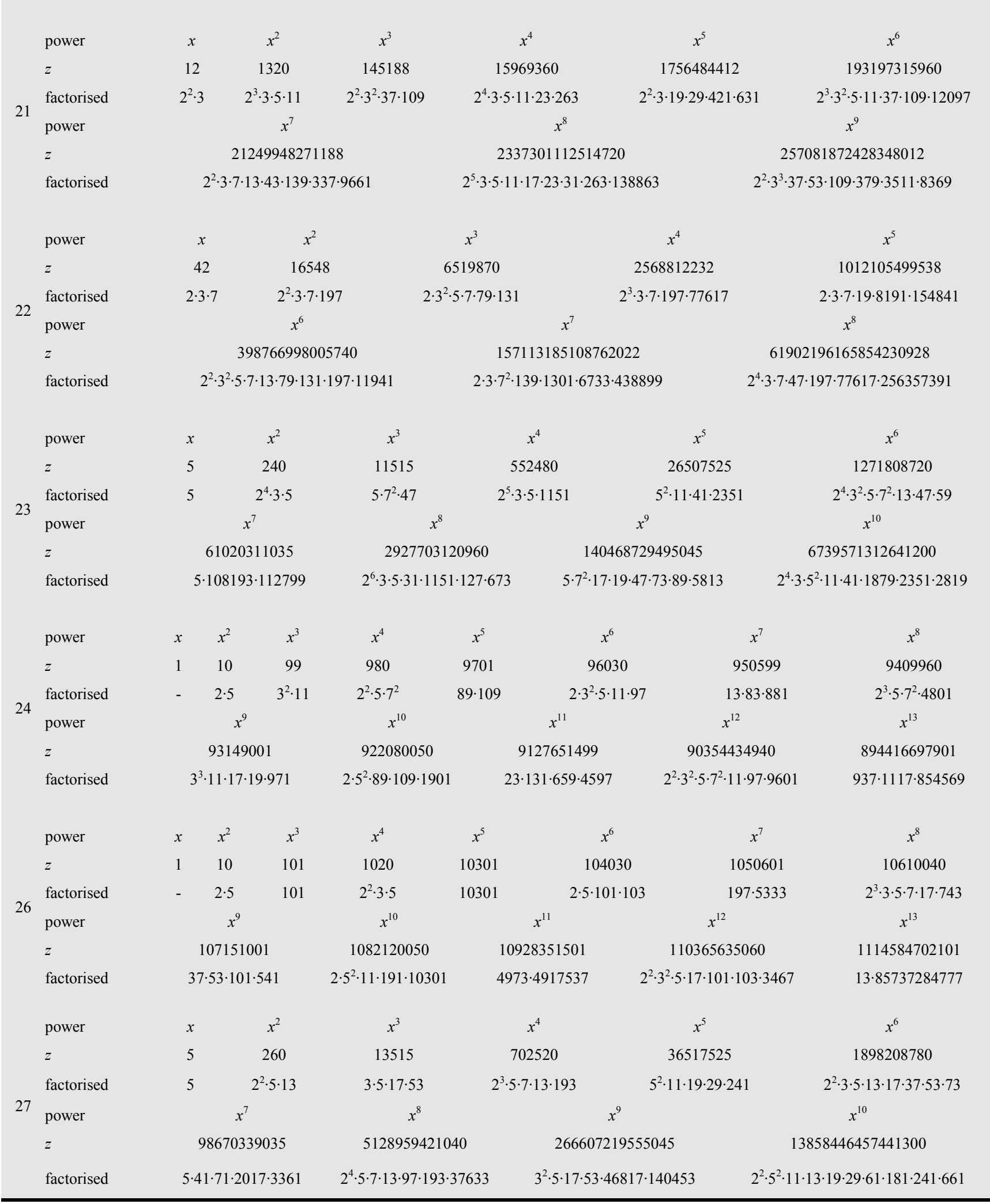

\section{Criteria for Series of Powers to Approach Integers}

A last phenomenon to consider in this paper is the approach of series of the integer powers of numbers to integers as the series progresses. As seen in table 6 the powers of the cycle factors more and more approximate integers as the powers increase. We thought that this could be unique to the root of a perfect square plus the root of a number adjacent to the perfect square $(y)$. The example used to investigate this scenario in table 9 is $2+\sqrt{5}$ (the 
cycle factor of $\sqrt{5}$ ). Testing this concept on a number 2 greater than the perfect square, 4 , it turned out to work for that number also ie $2+\sqrt{6}$, except that the approach to the integers was slower. Testing further numbers $(2+\sqrt{7}, 2+$ $\sqrt{8}$, etc) it was found that these series would approach integers as long as the root of a non-perfect square was within the bounds set by the next perfect square, $i e$ in this example not greater than $2+\sqrt{8} .2+\sqrt{9}$, of course is 5 and is not irrational, neither its powers. The power series of $2+$ $\sqrt{10}$ does not approach integers but is randomly spaced between the integers as the series progresses. However, the series of the powers of $3+\sqrt{10}$ (cycle factor of $i a \sqrt{10}$ ) approaches integers etc.

Table 9. The behavior of the decimal portion of powers of different numbers.

\begin{tabular}{|c|c|c|c|c|}
\hline & $+\sqrt{6}$ & $2+832040 / 196418$ & $2+\sqrt{5}$ & $2+\sqrt{5.5}$ \\
\hline & 4.4494897427831780981972841 & 236067977476606013705464876 & 4.236067977499789696409173669 & 1 245007870011714777280815 \\
\hline & 19.797958971132712392789136 & 3383903 & 704107100000 & 23 \\
\hline & 88.090815370097205767551113 & 76.01315561624838192003129769 & 76.01315561749642483895595237 & 82.04113789845500860244926349 \\
\hline & 391.95917942265424785578273 & & 22100600427009105014 & \\
\hline & 1744.0183484308114029582331 & 1364.000733100110488250780517 & 1364.000733137435857404797969 & \\
\hline & 7759.9917525685541075444980 & 5777.999826739992926535141491 & 5777.999826929728287760652380 & 6730.748307673310705400839606 \\
\hline & 34528.003707135839236094458 & 4476.00003991866180591544118 & 24476.0000408563490084 & 29246.50058420 \\
\hline & 153631.99833368046515946683 & 103681.9999858155737712118222 & 103681.9999903551243215502823 & 127082.1247983280001757005911 \\
\hline & 683584.00074899353911005623 & 139203.9999806432709863611507 & 439204.0000022768462946485368 & \\
\hline & 3041599.9996633350867591586 & 1860497.999897638847720127136 & 1860497.99999946250 & 2399416 \\
\hline & 13533568.000151327425256747 & 7881195.999525661735976614070 & & \\
\hline & 60217471.999931979874545304 & 3385281.99780738827807014617 & & \\
\hline & 267937024.00003057434869471 & 141422323.9899380878681459155 & & 196850 \\
\hline & 1192183039.9999862571438695 & & & \\
\hline & 5304606208.0000061772728672 & 2537720635.791668676418882018 & & \\
\hline & 23602790911.99999722 & 1074 & & \\
\hline & 105020376064.00000124806257 & 377806 & 45537 & \\
\hline & 467287086079.99999943900868 & 192900153598.9968943809622086 & & \\
\hline & 2079189096448.0000002521598 & & & \\
\hline 20 & 9251330557951.9999998866567 & 3461452807623.1145618 & & \\
\hline & 41163700424704.000000050947 & & & \\
\hline 22 & 183157462814719.99999997710 & & & \\
\hline$y^{23}$ & 814957252108288.00000001029 & 263115950924155.6805608021695 & & \\
\hline & 3626143934062591.9999999954 & & & \\
\hline & 1613449024046694 & & & \\
\hline$y^{26}$ & 71790248829992959.999999999 & 2000027372 & 20000273725560978.00000000000 & 38750250969527716.58666992188 \\
\hline, 27 & 319429975800905728.00000000 & 84722519057559915.99979103221 & 84722519070079276.00000000000 & 168377895861348399.4 \\
\hline & 1421300400863608832.0000000 & & & \\
\hline$y^{29}$ & & & & \\
\hline & 28138847021952204800.000000 & 6440026025322871098.089408896 & 6440026026380244498.000000000 & 13813914173412580918.87469482 \\
\hline & 125203511197921312768.00000 & 27280388019986160476.40395370 & 27280388024614569596.00000000 & 60024328718736468610.43615723 \\
\hline & 557091738835589660672.00000 & 115561578104599807363.8217762 & 115561578124838522882.0000000 & 260818186135064745820.0566711 \\
\hline & 2478773977738201268224.0000 & 489526700435556943452.2002600 & 489526700523968661124.0000000 & 1133309237618363686195.880920 \\
\hline
\end{tabular}

The series of an approximation to the cycle factor of $\sqrt{5}$, $832040 / 196418\left(j_{10} / j_{9}\right.$ of the $e$-series of triangles having triangle $2 / \sqrt{5} / 3$ as limit [1]) started approaching integers, but at $(2+832040 / 196418)^{8}$ the series was at its closest approach and then started drifting away again as the series progressed. Clearly the cycle factor must be correct and not approximated to have the series of powers continue approaching integers.

Then we wondered whether any square root in the defined bounds would approach integers as the power series continued? We tested this concept with $2+\sqrt{5.5}$. Its powers were randomly dispersed between integers with the 
progression of the powers.

The following rule seems to apply generally. A series ( $x$ $\left.+\sqrt{x^{2} \pm r}\right)^{m}$ will come closer and closer to integers as $m$ progresses from $1-\infty$ on condition that $r<(x+1)^{2}-x^{2}$ for the + condition and $r<x^{2}-(x-1)^{2}$ for the - condition, and $x$ and $r$ are positive integers. The equation $x^{2}=\left(n \times z^{2}\right) \pm 1$ does not apply to any other numbers but the ones which may be defined as $y=x+\sqrt{x^{2} \pm 1}, x$ being a positive integer.

\section{Conclusion}

In this paper it has been shown that cycle factors $(y)$ for series of right-angled triangles [1] that have a right-angled triangle with one irrational leg as the limit, are defined by $y$ $=x+\sqrt{x^{2} \pm 1}$ where $x$ is a positive integer. Clearly, as $x$ increases, $x+\sqrt{x^{2} \pm 1}$ will come closer and closer to an integer. Powers of the cycle factors also obey the same rule, ie $\left(x+\sqrt{x^{2} \pm 1}\right)^{\mathrm{m}}=x^{\prime}+\sqrt{x^{\prime 2} \pm 1}$ for any positive integer value of $m$. Therefore a series of powers of $x+\sqrt{x^{2} \pm 1}$ will approximate a positive integer closer and closer as the series progresses. It has been shown that the cycle factor of a root of $n$, that is not adjacent to a perfect square, neither is a perfect square, can be determined by finding the smallest natural number solution $(z)$ to the equation $x^{2}=\left(n \times z^{2}\right) \pm 1$ and $x$ is the integer portion of the cycle factor $(y)$. For $1-$ 100, each non-perfect square has such a solution. The powers of the cycle factors also adhere to the same equation, rendering the greater solutions $(z)$ to that equation. Prime factorising $z$ for the series of increasing powers, reveals discrete patterns in the repetition of the prime factors.

The following questions arise from this work.

- $\quad$ Do all the positive integers $(n)$ that are not perfect squares have a solution for the equation $x^{2}=\left(n \times z^{2}\right)$ \pm 1 ? The integers below 102 that are not perfect squares all have solutions to this equation.

- Why do the prime factors of $z$ in the equation $x^{2}=$ $\left(n \times z^{2}\right) \pm 1$ in the series of powers of cycle factors display such predictable behavior? Do these patterns apply to the greater prime factors also?

- Why does a series $\left(x+\sqrt{x^{2} \pm r}\right)^{m}$ come closer and closer to integers as $m$ progresses from $1-\infty$ on condition that $r<(x+1)^{2}-x^{2}$ for the positive condition and $r<x^{2}-(x-1)^{2}$ for the negative condition, and $x$ and $r$ are positive integers, but not to any other value of $r$, be it outside the range or non-integer?

- What determines when $x^{2}=\left(n \times z^{2}\right) \pm 1$ is +1 and especially when it is -1 ?

- When roots share cycle factors $(y)$, they are sometimes exactly the same, and sometimes one cycle factor is a power of the other. What determines whether they are the same or not, and when powers are involved, what determines which power?

These phenomena are being further investigated to procure a better understanding for their existence. Furthermore, the concept will be studied in terms of cubes and cube roots, and higher powers.

\section{References}

[1] MW Bredenkamp, Applied and Computational Mathematics, 2013, 2, 42-53.

[2] All rational right-angled triangles may be raised or reduced to a relatively prime right-angled triangle defined by a pair of positive integer indices $(i, j)$ where $i$ is an uneven number and $j$ is an even number and the even leg $(e)$, the uneven leg $(u)$ and the hypotenuse $(h)$ of the triangle are algebraically defined by the indices $(i, j)$ as follows.

$$
\begin{aligned}
& u=i^{2}+i j \\
& e=j^{2} / 2+i j \\
& h=i^{2}+i j+j^{2} / 2
\end{aligned}
$$

[3] MW Bredenkamp, Applied and Computational Mathematics, 2013, 2, 36-41. 